\title{
Reproducibility of exhaled breath condensate $\mathrm{pH}$ in chronic obstructive
} pulmonary disease

\author{
Z. Borrill, C. Starkey, J. Vestbo and D. Singh
}

ABSTRACT: Increasingly, exhaled breath condensate (EBC) is being used to sample airway fluid from the lower respiratory tract. EBC pH may be a biomarker of airway inflammation in chronic obstructive pulmonary disease (COPD). In this study, the reproducibility of EBC pH in COPD was investigated.

A total of 36 COPD patients and 12 healthy nonsmoking subjects participated in several investigations: duration of argon deaeration, within-sample variability, effect of freezing, leaving samples at room temperature, nose-peg use, within- (WD) and between-day (BD) variability. Analysis of repeated measurements was performed using the Bland-Altman method with limits of agreement (LOA; mean difference $\pm 2 \mathrm{SD}$ ). Wider LOA indicate greater variability.

EBC pH became significantly higher with argon deaeration for $\leqslant \mathbf{m i n}$. Variability during sample analysis was minimal; LOA of within-sample variability, freezing for 3 months and leaving at room temperature for $3 \mathrm{~h}$ were $-0.29-0.45,-0.37-0.42$ and $-0.13-0.09$, respectively. In contrast, variability due to nose-peg use (LOA -1.46-1.99), WD (LOA -1.50-2.48) and BD variability (LOA -2.52-3.02) were higher in COPD. In healthy nonsmoking subjects, nose-peg use (LOA -0.27-0.23), WD (LOA -0.33-0.40) and BD variability (LOA -0.46-0.44) were more reproducible.

In conclusion, the variability of exhaled breath condensate $\mathrm{pH}$ in chronic obstructive pulmonary disease patients is mainly due to changes in airway $\mathrm{pH}$ over time, which are not seen in healthy nonsmoking subjects. Reasons for these fluctuations in exhaled breath condensate $\mathrm{pH}$ are unclear and require further investigation.

\section{KEYWORDS: Chronic obstructive pulmonary disease, exhaled breath condensates}

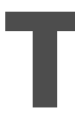
he use of exhaled breath condensate (EBC) to sample airway lining fluid from the lower respiratory tract is increasing. Acidification of EBC has been demonstrated in patients with asthma, chronic obstructive pulmonary disease (COPD) and bronchiectasis [1, 2]. The underlying pathophysiological processes that are responsible for the reduction in EBC $\mathrm{pH}$ in COPD patients are unclear. Nevertheless, EBC $\mathrm{pH}$ correlates with sputum neutrophilia, is reduced during exacerbations [2, 3], and, thus, may serve as a biomarker of disease severity.

The potential use of EBC $\mathrm{pH}$ as a biomarker in COPD patients may be limited by method reproducibility. Factors that may affect reproducibility include the following: 1) methodological issues during sample analysis, i.e. duration of argon deaeration, effect of freezing and time at room temperature; 2) methodological issues during sample collection, e.g. differences due to nose-peg use or the length of collection time; and
3) variability between samples collected at different times from the same subject, i.e. within(WD) and between-day (BD) variability. These issues have not been thoroughly investigated in COPD patients, and no formal guideline has yet been proposed.

The current authors investigated the reproducibility of EBC $\mathrm{pH}$ in COPD patients. The variability attributable to methodological issues during sample collection and analysis was assessed. Additionally, WD and BD variability in COPD patients were determined and compared with data from healthy subjects.

\section{METHODS}

\section{Subjects} according to current criteria [4], and 12 healthy nonsmoking volunteers took part in the study (table 1). Subjects were excluded if they had experienced an exacerbation of COPD or
A total of 36 patients with COPD, diagnosed

\section{AFFILIATIONS}

Medicines Evaluation Unit, North West Lung Centre, Wythenshawe Hospital, Manchester, UK.

CORRESPONDENCE

Z. Borrill

Wythenshawe Hospital North West Lung Centre Manchester M23 9LT UK

Fax: 441612912243

E-mail: zborrill@meu.org.uk

Received:

July 212004

Accepted after revision:

October 202004 


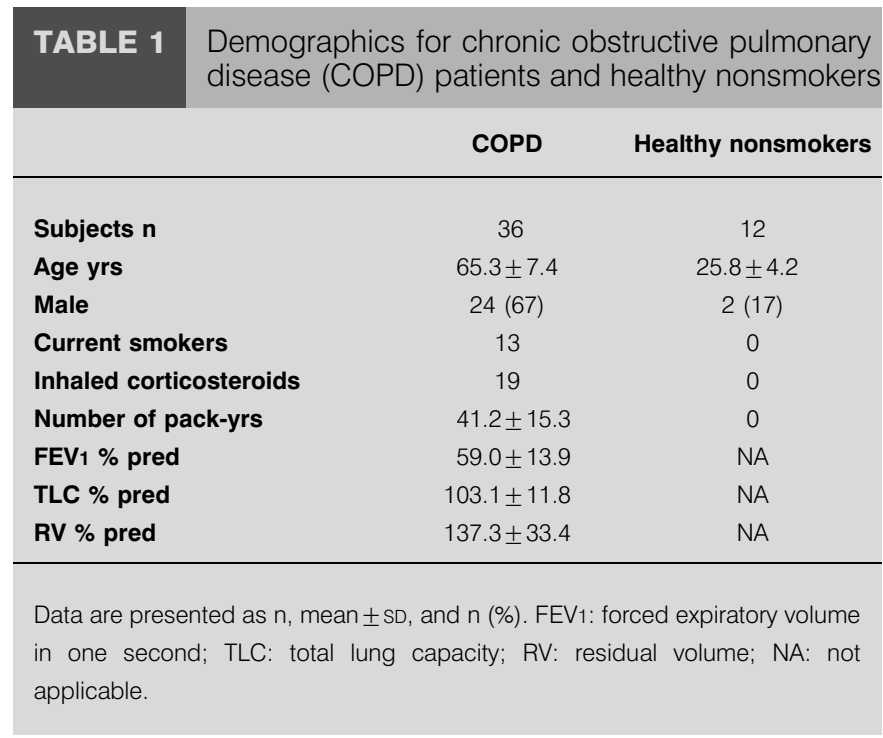

respiratory tract infection within the last 4 weeks. COPD patients abstained from short- and long-acting bronchodilators for 6 and $12 \mathrm{~h}$, respectively, and from tiotropium for $24 \mathrm{~h}$. Caffeine and cigarettes were withheld from all subjects for $2 \mathrm{~h}$ prior to EBC collection. All subjects provided written informed consent and the local ethics committee approved the study.

\section{Exhaled breath condensate collection}

EBC collection was carried out using the following standard method, and any deviations from this are detailed separately. EBC was collected during tidal breathing for $10 \mathrm{~min}$ (EcoScreen; Jaeger, Hoechberg, Germany) without a nosepeg. Subjects were instructed to breathe normally through their mouth and to temporarily discontinue collection if they needed to swallow saliva or cough. Samples were aliquoted into separate $200-\mu \mathrm{L}$ tubes. Argon gas was passed over the sample at $2 \mathrm{~L} \cdot \mathrm{min}^{-1}$ for $10 \mathrm{~min}$ to achieve deaeration, after which $\mathrm{pH}$ was measured using a pH 210 meter (Hanna instruments, Leighton Buzzard, UK) with a Biotrode electrode (Hamilton, Remo, NV, USA). Amylase was measured in 30 samples using Infinity amylase liquid assay system (Thermo Electron, Grenoble, France; lower limit of detection $50 \mathrm{U} \cdot \mathrm{L}^{-1}$ ) and was found to be undetectable.

\section{Duration of argon deaeration}

EBC was collected from eight COPD patients. Each sample was divided into six aliquots and frozen at $-80^{\circ} \mathrm{C}$ for 2 weeks before analysis. After defrosting, the six aliquots were deaerated for 0 , $1,2,5,10$ and $15 \mathrm{~min}$ prior to $\mathrm{pH}$ measurement.

\section{Within-sample variation}

Samples collected from 10 COPD patients were divided into two aliquots and analysed immediately.

\section{Effect of freezing}

Samples from 15 COPD patients were divided into three aliquots. One aliquot was analysed immediately after collection and the remaining two after freezing at $-80^{\circ} \mathrm{C}$ for 2 weeks and 3 months, respectively.

\section{Length of time at room temperature before analysis}

EBC was collected from five COPD patients. Each sample was divided into four aliquots and analysed without freezing. The first aliquot was analysed immediately and the others after standing at room temperature for $15 \mathrm{~min}, 1 \mathrm{~h}$ and $3 \mathrm{~h}$.

\section{Differences in $\mathbf{p H}$ during sample collection}

The purpose of this experiment was to assess the differences in $\mathrm{pH}$ during initial sample collection (e.g. the first $3 \mathrm{~min}$ ) compared with later time periods during a collection. EBC was collected from 11 COPD patients for $9 \mathrm{~min}$. At 3-min intervals, the sample collected was removed and frozen at $-80^{\circ} \mathrm{C}$ (aliquots $1-3$ ) for analysis 2 weeks later.

Effect of nose-peg use, within- and between-day variability EBC was collected from 15 COPD patients and 12 healthy volunteers without a nose-peg (collection without a nose-peg is referred to as test 1) and using a nose-peg, in a random order. Further samples were collected without a nose-peg $1 \mathrm{~h}$ and 1 week later (these collections are referred to as test 2 and test 3 , respectively). All samples were frozen at $-80^{\circ} \mathrm{C}$ for 2 weeks before analysis.

\section{Statistical analysis}

Pearson's correlation coefficient was used to assess the relationship between forced expiratory volume in one second (FEV1) and $\mathrm{pH}$ in COPD. An unpaired t-test was used to assess the effect of current smoking and the use of inhaled corticosteroids in COPD, and to compare EBC $\mathrm{pH}$ in COPD patients and healthy volunteers. The first $\mathrm{pH}$ measurement obtained from each subject by the standard collection and analysis method was used for all of these comparisons. Duration of argon deaeration was assessed using a paired $\mathrm{t}$ test, with Bonferoni correction for multiple comparisons. pValues of $<0.05$ were considered statistically significant.

The data for repeated measurements are presented as mean (95\% confidence interval (CI)). Repeated measurements were also analysed using the Bland-Altman method, which allows assessment of the limits of agreement. These limits provide an estimate of the variability expected from individual measurements, as $95 \%$ of repeated measurements lie within the limits of agreement [5].

\section{RESULTS}

A wide range of EBC $\mathrm{pH}$ values (4.75-7.91) was observed in COPD patients. The mean $(95 \% \mathrm{CI}) \mathrm{pH}$ was significantly lower in COPD patients (6.97 (6.65-7.29)) compared with healthy volunteers (7.61 (7.52-7.70); $\mathrm{p}=0.03)$. The mean EBC $\mathrm{pH}$ of those COPD patients taking inhaled corticosteroids was numerically higher (7.22 (6.87-7.58)) than those not taking inhaled corticosteroids (6.69 (6.15-7.22)), although this difference was not statistically significant $(\mathrm{p}=0.11)$. EBC $\mathrm{pH}$ was lower in current smokers with COPD (6.66 (6.12-7.21)) compared with ex-smokers (7.12 (6.75-7.54)), but, again, the difference was not statistically significant $(p=0.16)$. There was no correlation between FEV1 and EBC $\mathrm{pH}(\mathrm{r}=-0.09 ; \mathrm{p}=0.61)$ in COPD patients (fig. 1).

\section{Duration of argon deaeration}

EBC $\mathrm{pH}$ became significantly less acidic $(\mathrm{p}<0.05)$ with argon deaeration up to $5 \mathrm{~min}$ (fig. 2). After $5 \mathrm{~min}$, there was no 


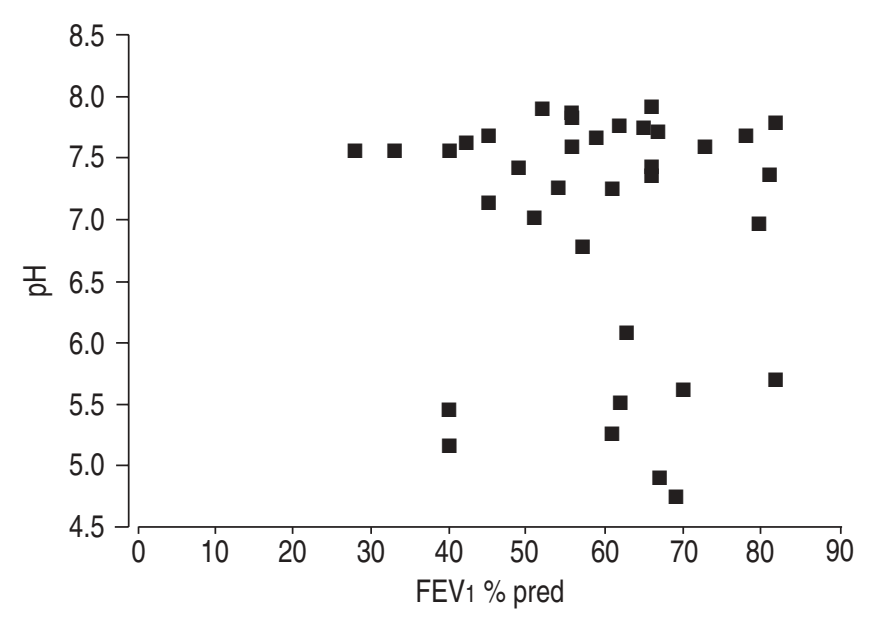

FIGURE 1. Correlation of forced expiratory volume in one second (FEV 1 ) versus exhaled breath condensate $\mathrm{pH}$ in chronic obstructive pulmonary disease patients $(n=36) . r=-0.09$.

significant change in $\mathrm{pH}$. Argon caused an overall increase in $\mathrm{pH}$ of approximately one log order.

\section{Within-sample variation}

The $\mathrm{pH}$ readings of two aliquots from the same sample showed a mean difference of 0.08 . The limits of agreement were -0.29 and 0.45 (fig. 3).

\section{Effect of freezing, length of time at room temperature before analysis and differences in $\mathrm{pH}$ during sample collection}

The mean $\mathrm{pH}$ values obtained after immediate analysis (7.03 (6.50-7.57)) and after freezing for 2 weeks (7.09 (6.56-7.63)) and 3 months (7.06 (6.51-7.60)) were similar. The mean $\mathrm{pH}$ values obtained from samples analysed after standing at room temperature for $15 \mathrm{~min} \quad(7.56 \quad(7.36-7.76)), 1 \mathrm{~h} \quad(7.56$ (7.41-7.70)) and $3 \mathrm{~h}(7.55$ (7.36-7.74)) were comparable with those analysed immediately (7.57 (7.39-7.75)). The limits of

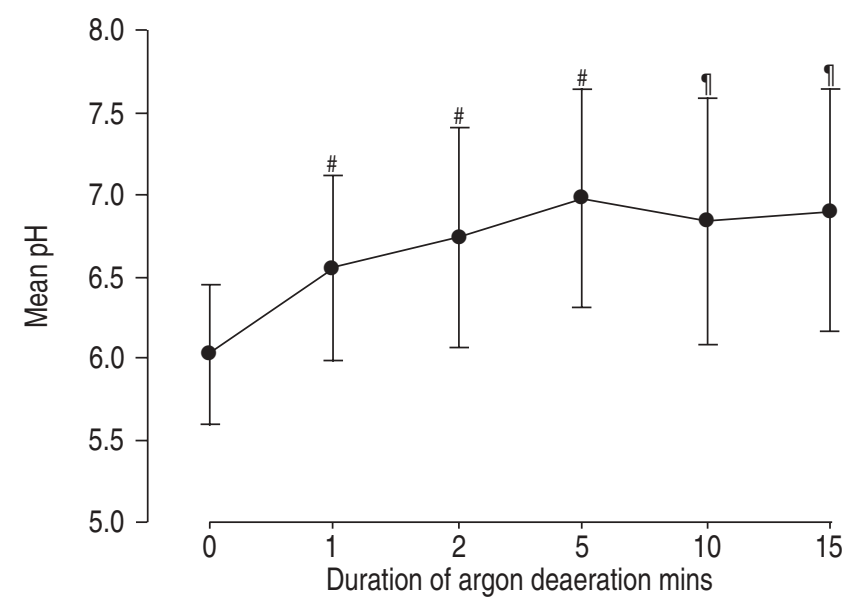

FIGURE 2. Change in exhaled breath condensate $\mathrm{pH}$ after increasing duration of argon deaeration in samples from eight chronic obstructive pulmonary disease patients. ${ }^{*}: p<0.05 ;{ }^{\circ}: p>0.05$ compared with shorter duration time.

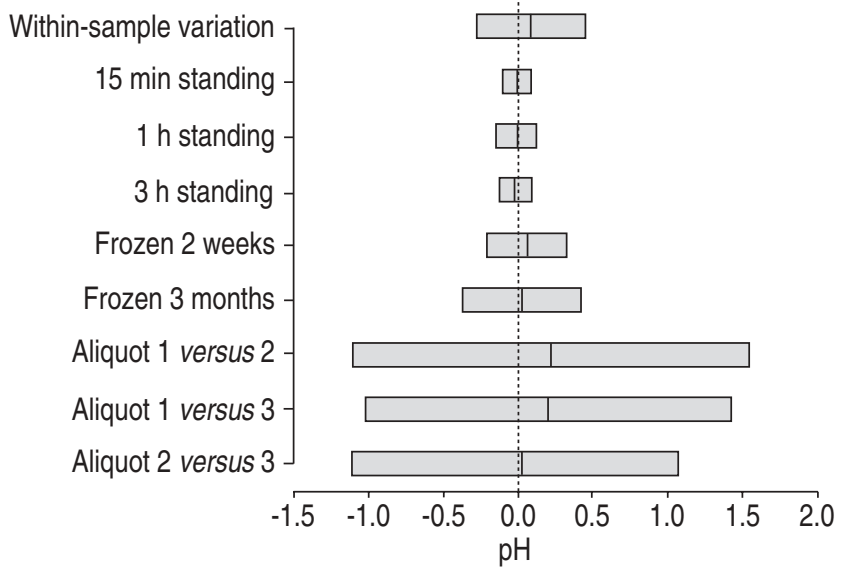

FIGURE 3. Limits of agreement for within-sample variability $(n=10)$, length of time at room temperature before analysis $(n=5)$, effect of freezing $(n=15)$ and differences in $\mathrm{pH}$ during sample collection in chronic obstructive pulmonary disease patients $(n=11)$. Vertical lines indicate mean differences.

agreement for these experiments were no greater than the within-sample variation (fig. 3). The mean $\mathrm{pH}$ values from the three samples collected over 3-min intervals during a 9-min collection period were similar: aliquot 1, 7.21 (6.84-7.58); aliquot 2, 7.43 (7.24-7.62); and aliquot 3, 7.41 (7.16-7.65). However, the corresponding limits of agreement showed a greater degree of variability compared to within-sample variation (fig. 3).

Effect of nose-peg use, within- and between-day variability In healthy subjects, the mean $\mathrm{pH}$ values of samples obtained without a nose-peg (7.61 (7.52-7.70)) and using a nose-peg (7.59 (7.47-7.70)) were similar. The mean values of samples taken $1 \mathrm{~h}$ and 1 week later without a nose-peg were 7.62 (7.52$7.71)$ and 7.59 (7.49-7.68), respectively. In COPD patients, the mean $\mathrm{pH}$ values using a nose-peg (6.95 (6.40-7.50)) and without a nose-peg $(6.69(6.13-7.24))$ were similar. The mean values after $1 \mathrm{~h}$ and 1 week without a nose-peg were 7.18 (6.70-7.65) and 6.94 (6.38-7.49). The limits of agreement for nose-peg use, as well as WD and BD variability in healthy subjects, were relatively small (fig. 4). In contrast, the limits of agreement for these parameters were much greater in COPD patients. Nose-peg use and WD variability were similar in COPD patients, while BD variability had the widest limits of agreement (figs 4 and 5). The limits of agreement were similar in ex-smokers and current smokers with COPD (data not shown). There was no correlation between the degree of variability and FEV1, age or corticosteroid use in COPD patients.

\section{DISCUSSION}

The reproducibility of EBC $\mathrm{pH}$ measurements in COPD patients has not previously been thoroughly investigated. It was found that methodological issues during sample analysis (duration of argon deaeration, effect of freezing and time at room temperature) only cause minor variations in $\mathrm{pH}$ values. In contrast, methodological issues during sample collection (use of a nose-peg and the length of collection time) cause greater variability. It has also been shown that the WD and BD 


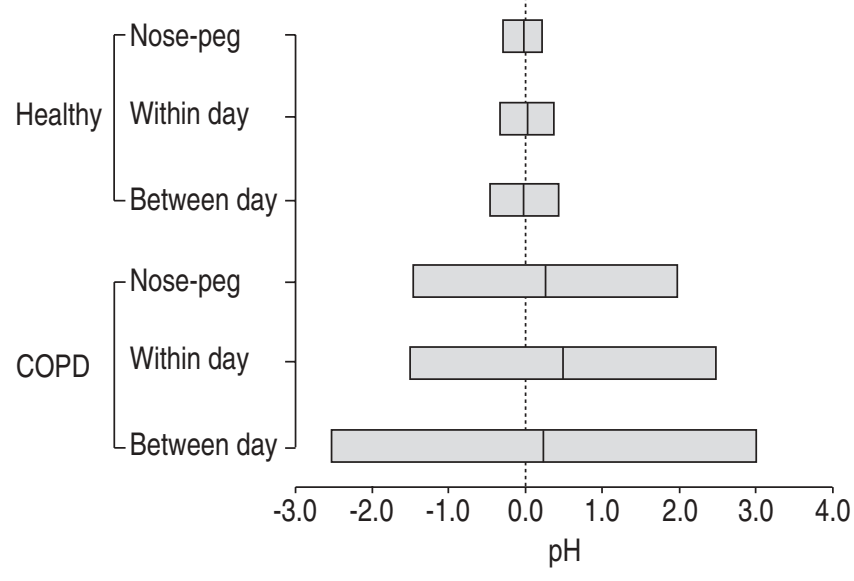

FIGURE 4. Limits of agreement for the effect of nose-peg use, within-day variability (without a nose-peg) and between-day variability (without a nose-peg) in healthy subjects $(n=12)$ and chronic obstructive pulmonary disease (COPD) patients $(n=15)$. Vertical lines indicate mean differences.

variability of EBC $\mathrm{pH}$ in COPD can be considerable, and is greater than that observed in healthy volunteers. In COPD patients, this indicates that there are changes in EBC $\mathrm{pH}$ over time that do not occur in healthy subjects.

Repeated measurements in the paper were analysed by the following two methods: 1) mean (95\% CI); and 2) the BlandAltman method to determine the limits of agreement. The calculation of mean (95\% CI) values allows assessment of group variability. In contrast, the Bland-Altman method provides an estimate of the variability between individual measurements [5]. For example, limits of agreement can be used to estimate the variation that can be expected when EBC $\mathrm{pH}$ samples are obtained from the same individual on 2 separate days, i.e. BD variation. KosTIKAS et al. [2] reported the reproducibility of $\mathrm{EBC} \mathrm{pH}$ on 2 consecutive days in COPD patients. However, only the mean data was presented and, therefore, there was no estimate of the variability between individual samples.

One of the most important experiments in this paper was the assessment of within-sample variability. This provided an estimate of method variation during repeated measurements of the same sample. These data were a guide to the minimum variability to be expected in all of the other experiments involving repeated measurements, e.g. if freezing changed EBC $\mathrm{pH}$ in the same sample, the effect should be greater than within-sample variation. Similarly, to assess WD or BD variability in the same subjects (i.e. the effect of time), withinsample variation was used to control for the variability that was attributable to the process of sample analysis.

There are no published data regarding methodological aspects of the analysis of EBC $\mathrm{pH}$ in samples from COPD patients. Fundamentally, samples are often frozen and argon deaeration is performed prior to analysis, but there are no data in COPD patients to support this. Additionally, it is often recommended that samples be analysed immediately if left at room temperature, although, again, there are no published data to support this recommendation. These methodological issues
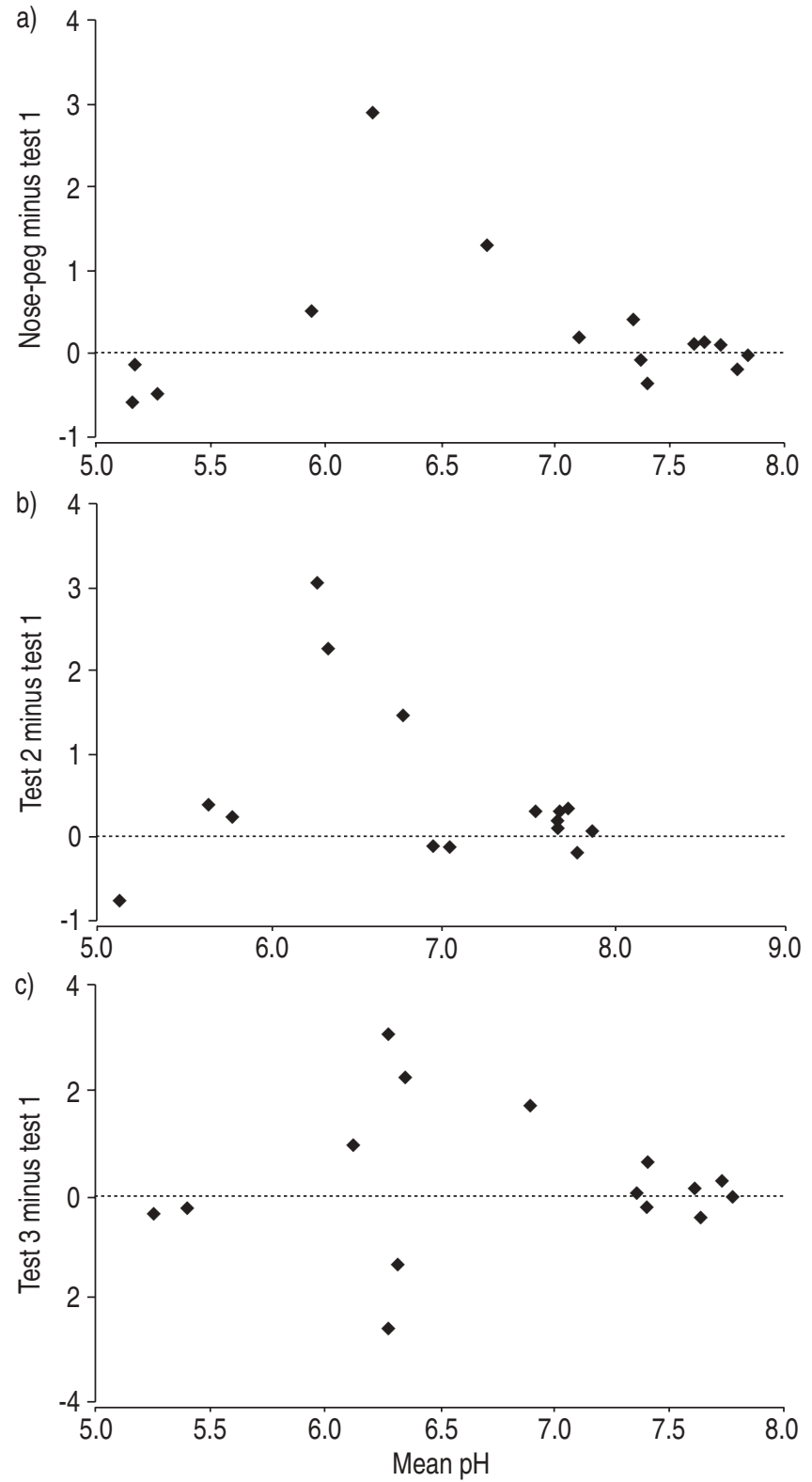

FIGURE 5. Bland-Altman plots for a) variability due to nose-peg use, b) withinday variability (without a nose-peg) and c) between-day variability (without a nosepeg) in chronic obstructive pulmonary disease patients $(n=15)$.

were investigated in the current study. Freezing samples at $-80^{\circ} \mathrm{C}$ for $\leqslant 3$ months caused variability similar to withinsample variation. This suggests that freezing has no effect on EBC $\mathrm{pH}$ in samples from COPD patients. The current results are similar to those reported in healthy volunteers where the $\mathrm{pH}$ remained stable after freezing for $\leqslant 2$ yrs [6]. It was also found that leaving samples standing at room temperature for $\leqslant 3 \mathrm{~h}$ before analysis caused minor variability that was of similar magnitude to within-sample variability, indicating that time at room temperature up to $3 \mathrm{~h}$ has no effect on EBC $\mathrm{pH}$ in samples from COPD patients.

Previous studies of EBC $\mathrm{pH}$ have used argon deaeration for the removal of $\mathrm{CO}_{2}$ prior to $\mathrm{pH}$ measurement $[1,2,6,7]$. This 
process causes $\mathrm{CO}_{2}$ within the sample to diffuse out along a concentration gradient until equilibrium occurs. Changes in $\mathrm{pH}$ were observed for the first $5 \mathrm{~min}$ of this procedure. Continued deaeration after this time resulted in no further change in $\mathrm{pH}$. It has been suggested that deaeration should be performed until $\mathrm{pH}$ stabilisation. The current data indicate that stabilisation in COPD patients occurs after $5 \mathrm{~min}$, and this duration of deaeration is recommended. The magnitude of change in EBC pH after argon deaeration was approximately one, which is similar to a recent report in patients with cough [7]. The current method of deaeration involved passing argon over rather than through the EBC sample [1, 2]. This was adopted due to the small volumes available in the present study. The current samples reached a stable $\mathrm{pH}$ within $5 \mathrm{~min}$, indicating that this method is effective in achieving the same degree of deaeration. However, it is possible that larger samples may require argon gas to be bubbled through the sample or a longer duration of argon deaeration to achieve a stable $\mathrm{pH}$, and further studies are needed to address this issue. Furthermore, the possibility of incomplete removal of $\mathrm{CO}_{2}$ by argon deaeration exists, and measurement of $\mathrm{CO}_{2}$ in future EBC samples would clarify this issue.

EBC samples are usually collected over $10 \mathrm{~min}$, but there are no data to support this practice. The limits of agreement for consecutive 3-min collections in COPD patients were greater than within-sample variability, indicating that there are changes in airway $\mathrm{pH}$ within a $10-$ min collection period. The reasons for this are unclear and require further investigation.

In COPD patients, the limits of agreement for nose-peg use, $\mathrm{WD}$ and $\mathrm{BD}$ variability were all greater than within-sample limits of agreement. This suggests that nose-peg use alters EBC $\mathrm{pH}$. However, the limits of agreement for nose-peg use and WD variability (i.e. two samples collected without a nose-peg) were similar. It could, therefore, be argued that the variability observed with a nose-peg is due to changes in airway $\mathrm{pH}$ over time, rather than the nose-peg itself.

In COPD patients, there were changes in airway $\mathrm{pH}$ over time, with greater differences observed as the length of time between samples was increased. In contrast, the WD and BD variability of EBC pH in healthy subjects was lower. This suggests that airway $\mathrm{pH}$ fluctuates in COPD patients. The mechanisms of airway $\mathrm{pH}$ control are poorly understood and require further study. The COPD patients and healthy volunteers in this study were not age matched. However, $\mathrm{pH}$ variability in healthy subjects in this study was similar to reported data [6], so it is likely that a true estimate of this value was obtained.

Several COPD patients had recorded $\mathrm{pH}$ values of $<5$, which changed to $>7$ after $1 \mathrm{~h}$ or 1 week. EBC $\mathrm{pH}$ values of $\leqslant 5$ have been reported previously in healthy subjects, as well as patients with lung disease $[1,2,6]$. However, this is the first report to demonstrate that these values do not remain constantly low. Airway inflammation, oxidative stress and bacterial colonisation are known to affect EBC $\mathrm{pH}$ [2]. However, their relative contribution to variations in $\mathrm{pH}$ over time is unknown. Furthermore, it is possible that other factors, such as gastro-oesophageal reflux, diurnal variation or respiratory pattern, may contribute to this variability, and further investigation is required. This variability cannot be attributed to errors in the sample analysis, as similar results were not observed when within-sample variability was assessed.

Potentially, EBC is derived from the oral cavity, oropharynx, tracheobronchial tree and alveoli, and their relative contributions to EBC $\mathrm{pH}$ is unclear. EBC contains ammonia, which is predominantly derived from the mouth [8]. However, it has been demonstrated that EBC samples taken directly before intubation have the same $\mathrm{pH}$ as samples taken from the endotracheal tube [6], indicating that EBC $\mathrm{pH}$ reflects airway $\mathrm{pH}$ and that oral contribution to the sample is less clinically important. Similarly, it is unlikely that salivary contamination contributed to EBC $\mathrm{pH}$ variability, as there was a failure to detect salivary amylase in 30 EBC samples, which is in agreement with other studies $[1,7]$.

COPD patients were asked to refrain from smoking for $\geqslant 2 \mathrm{~h}$ prior to EBC collection. Similar variability was observed in COPD ex-smokers and current smokers, indicating that current smoking is not a major determinant of variability. Similarly, no relationship was found between the degree of variability and FEV1, age or corticosteroid use.

Unlike KOSTIKAS et al. [2], the current authors found no correlation between FEV1 and EBC pH. However, a statistically nonsignificant trend towards reduced EBC $\mathrm{pH}$ in steroid naïve patients and ex-smokers was found. The current study was not statistically powered to detect a relationship between FEV1, smoking or steroid use and EBC $\mathrm{pH}$, and it is suggested that a larger study is needed to address these issues. Furthermore, a recent study has shown dissociation between airflow limitation and airway inflammation in COPD, suggesting that, if EBC $\mathrm{pH}$ was related to airway inflammation, it does not necessarily follow that a relationship to lung function will exist [9].

In summary, several methodological issues regarding exhaled breath condensate $\mathrm{pH}$ measurement in chronic obstructive pulmonary disease patients have been clarified. Specifically, exhaled breath condensate samples can be left standing at room temperature for $\leqslant 3 \mathrm{~h}$ and be frozen at $-80{ }^{\circ} \mathrm{C}$ for $\leqslant 3$ months without any change in $\mathrm{pH}$. Furthermore, stabilisation of exhaled breath condensate $\mathrm{pH}$ is achieved after argon deaeration for $5 \mathrm{~min}$. It has also been demonstrated that exhaled breath condensate $\mathrm{pH}$ measurements change over time in chronic obstructive pulmonary disease patients, and further work is required to investigate the reasons for this phenomenon.

\section{REFERENCES}

1 Hunt JF, Fang K, Malik R, et al. Endogenous airway acidification. Implications for asthma pathophysiology. Am J Respir Crit Care Med 2000; 161: 694-699.

2 Kostikas K, Papatheodorou G, Ganas K, Psathakis K, Panaagou P, Loukides S. $\mathrm{pH}$ in expired breath condensate of patients with inflammatory airway diseases. Am J Respir Crit Care Med 2002; 165: 1364-1370.

3 Antczak A, Gorski P. Endogenous airway acidification and oxidant overload in infectious exacerbation of COPD. Am J Respir Crit Care Med 2001; 163: 725A. 
4 National Collaborating Centre for Chronic Conditions. Chronic obstructive pulmonary disease. National clinical guideline on management of chronic obstructive pulmonary disease in adults in primary and secondary care. Thorax 2004; 59: Suppl. 1, 1-232.

5 Bland JM, Altman DG. Statistical methods for assessing agreement between two methods of clinical measurement. Lancet 1986; 1: 307-310.

6 Vaughan J, Ngamtrakulpanit L, Pajewski TN, et al. Exhaled breath condensate $\mathrm{pH}$ is a robust and reproducible assay of airway acidity. Eur Respir J 2003; 22: 889-894.
7 Niimi A, Nguyen LT, Usmani O, Mann B, Chung KF. Reduced $\mathrm{pH}$ and chloride levels in exhaled breath condensate of patients with chronic cough. Thorax 2004; 59: 608-612.

8 Effros RM, Biller J, Foss B, et al. A simple method for estimating respiratory solute dilution in exhaled breath condensates. Am J Respir Crit Care Med 2003; 168: 1500-1505.

9 Lapperre TS, Snoeck-Stroband JB, Gosman MME, et al. Dissociation of lung function and airway inflammation in chronic obstructive pulmonary disease. Am J Respir Crit Care Med 2004; 170: 499-504. 